\title{
Persistence and Extinction of a Stochastic Modified Bazykin Predator-Prey System with Lévy Jumps
}

\author{
Zhangzhi Wei, ${ }^{1,2}$ Zheng Wu, ${ }^{1}$ Ling Hu $\mathbb{D}^{1},{ }^{1}$ and Lianglong Wang ${ }^{1}{ }^{1}$ \\ ${ }^{1}$ School of Mathematical Sciences, Anhui University, Hefei 230601, China \\ ${ }^{2}$ School of Mathematics and Statistics, Suzhou University, Suzhou 234000, China \\ Correspondence should be addressed to Lianglong Wang; wangll@ahu.edu.cn
}

Received 7 December 2017; Accepted 12 March 2018; Published 29 April 2018

Academic Editor: Jeffrey Neugebauer

Copyright (c) 2018 Zhangzhi Wei et al. This is an open access article distributed under the Creative Commons Attribution License, which permits unrestricted use, distribution, and reproduction in any medium, provided the original work is properly cited.

\begin{abstract}
This paper is devoted to the dynamics of a stochastic modified Bazykin predator-prey system with Lévy jumps. First, we show that the system has a unique global positive solution and give some properties of solutions. Then, some sufficient conditions for persistence and extinction are derived by Itô formula and some inequalities on stochastic analysis. At last, some simulations are provided to check the main results.
\end{abstract}

\section{Introduction}

In population biology, construction of models and relevant qualitative analysis are popular fields $[1,2]$. In the last few decades, many predator-prey models with functional and numerical responses have been proposed and studied extensively. Particularly, ratio-dependent functional response is common in some classical literature [3-5].

Alexeev and Bazykin firstly proposed a Bazykin system [1]

$$
\begin{aligned}
& \dot{x}(t)=x(t)\left(a-b x(t)-\frac{c y(t)}{1+A x(t)}\right), \\
& \dot{y}(t)=y(t)\left(-g-h y(t)+\frac{f x(t)}{1+A x(t)}\right),
\end{aligned}
$$

where $x(t)$ and $y(t)$ are the size of prey and predator at time t. $a, b, c, f, g$, and $m$ are positive constants (some details refer to [1]).

Considering the ratio effect in hunting process of predation, Haque built a modified Bazykin system [2]

$$
\begin{aligned}
& \dot{x}(t)=x(t)\left(a-b x(t)-\frac{c y(t)}{y(t)+A x(t)}\right), \\
& \dot{y}(t)=y(t)\left(-g-h y(t)+\frac{f x(t)}{y(t)+A x(t)}\right),
\end{aligned}
$$

of which dynamical behavior near equilibrium point and bifurcation are observed. System (2) is more reasonable and many researchers began to pay more attention on it. Its generalized forms have been investigated and a lot of results were obtained [6-8].

However, in the natural world, environmental noise is everywhere, and the growth rate of the populations is not constants. In this case, many systems are described by stochastic differential equations driven by Brownian motion of the actual research. For example, [9] studied the following stochastic modified Bazykin system:

$$
\begin{aligned}
d x(t)= & x(t)\left(a-b x(t)-\frac{c y(t)}{y(t)+A x(t)}\right) d t \\
& +\alpha x(t) d w_{1}(t), \\
d y(t)= & y(t)\left(-g-h y(t)+\frac{f x(t)}{y(t)+A x(t)}\right) d t \\
& +\beta y(t) d w_{2}(t),
\end{aligned}
$$

where $w_{1}(t)$ and $w_{2}(t)$ are two independent Wiener processes defined on a complete probability space $\left(\Omega, \mathscr{F},\left\{\mathscr{F}_{t}\right\}_{t \geq 0}, P\right)$ and $\alpha$ and $\beta$ stand for the level of the white noises. Some sufficient conditions for persistence and extinction are obtained. 
Recently, the stochastic differential equation driven by jump has drawn more and more researchers' attention [1017]. This is mainly according to sudden perturbation of environment, such as toxic contamination of water, torrential flood, and hurricane. Motivated by the above, this paper considers the following stochastic modified Bazykin system with Lévy jumps:

$$
\begin{aligned}
d x(t)= & x\left(t^{-}\right)\left(a-b x(t)-\frac{c y(t)}{y(t)+A x(t)}\right) d t \\
& +\alpha x(t) d w_{1}(t) \\
& +\int_{Z} \gamma(u) x\left(t^{-}\right) \widetilde{N}(d t, d u), \\
d y(t)= & y\left(t^{-}\right)\left(-g-h y(t)+\frac{f x(t)}{y(t)+A x(t)}\right) d t \\
& +\beta y(t) d w_{2}(t)
\end{aligned}
$$

$$
+\int_{Z} \eta(u) y\left(t^{-}\right) \widetilde{N}(d t, d u)
$$

where $x\left(t^{-}\right), y\left(t^{-}\right)$represent the left limit of $x(t), y(t)$. $\widetilde{N}(d t, d u)=N(d t, d u)-\lambda(d u) d t, N$ is a Poisson random measure, and $\lambda$ is the characteristic measure of $N$ on a measurable subset $Z \subset R^{+}=(0,+\infty)$ with $\lambda(Z)<+\infty$.

The rest of this paper is organized as follows. In Section 2, some properties of positive solutions to system (4) are discussed. In Section 3, the main results for persistence and extinction are given. Finally, the simulation results show the validity of our results.

\section{Properties of Positive Solutions}

Throughout this paper, we require that $w_{1}(t), w_{2}(t)$, and $N$ are independent and

$$
\begin{aligned}
& \left(H_{1}\right) \quad \max \left\{\int_{Z}(\gamma(u))^{p} \lambda(d u), \int_{Z}(\eta(u))^{p} \lambda(d u)\right\}<+\infty, \quad p>0 \\
& \left(H_{2}\right) \quad \max \left\{\int_{Z}(\ln (1+\gamma(u)))^{2} \lambda(d u), \int_{Z}(\ln (1+\eta(u)))^{2} \lambda(d u)\right\}<+\infty
\end{aligned}
$$

with $\min \{1+\gamma(u), 1+\eta(u)\}>0, u \in Z$.

First, we present the global existence of positive solutions.

Lemma 1. For any given value $\left(x_{0}, y_{0}\right) \in R_{+}^{2}$, system (4) has a unique positive solution $X(t)=(x(t), y(t))$ on $t \geq 0$ and the solution will be in $R_{+}^{2}$ a.s. (almost surely).

Proof. Let $u(t)=\ln x(t), v(t)=\ln y(t)$, then we consider the following system:

$$
\begin{gathered}
d u(t)=\left[a-b e^{u(t)}-\frac{c e^{v(t)}}{e^{v(t)}+A e^{u(t)}}-\frac{\alpha^{2}}{2}\right. \\
\left.-\int_{Z}(\gamma(u)-\ln (1+\gamma(u))) \lambda(d u)\right] d t \\
+\alpha d w_{1}(t)+\int_{Z} \ln (1+\gamma(u)) \widetilde{N}(d t, d u), \\
d v(t)=\left[-g-h e^{v(t)}+\frac{f e^{u(t)}}{e^{v(t)}+A e^{u(t)}}-\frac{\beta^{2}}{2}\right. \\
\left.-\int_{Z}(\eta(u)-\ln (1+\eta(u))) \lambda(d u)\right] d t \\
+\beta(t) d w_{2}(t)+\int_{Z} \ln (1+\eta(u)) \widetilde{N}(d t, d u)
\end{gathered}
$$

with initial date $u(0)=\ln x_{0}, v(0)=\ln y_{0}$ on $t \geq 0$. Since the coefficients of system (6) are locally Lipschitz continuous, then there is a unique local solution on $[0, T]$ a.s., where $T$ is blow-up time. Then $x=e^{u(t)}, y=e^{v(t)}$ is the unique positive local solution to system (4) with initial data $x_{0}>0, y_{0}>0$. We will show that $T=+\infty$, and this mean the solution is global.

Consider the following equations:

$$
\begin{aligned}
d X_{1}(t)= & X_{1}(t)\left(a-c-b X_{1}(t)\right) d t+\alpha X_{1}(t) d w_{1}(t) \\
& +\int_{Z} X_{1}(t) \gamma(u) \widetilde{N}(d t, d u), \\
X_{1}(0)= & x_{0}, \\
d X_{2}(t)= & X_{2}(t)\left(a-b X_{2}(t)\right) d t+\alpha X_{2}(t) d w_{1}(t) \\
& +\int_{Z} X_{2}(t) \gamma(u) \widetilde{N}(d t, d u), \\
X_{2}(0)= & x_{0}, \\
d Y_{1}(t)= & Y_{1}(t)\left[-g+\frac{f}{A}-\left(h+\frac{f}{A^{2} X_{1}(t)}\right) Y_{1}(t)\right] d t \\
& +\beta Y_{1}(t) d w_{2}(t) \\
& +\int_{Z} Y_{1}(t) \eta(u) \widetilde{N}(d t, d u),
\end{aligned}
$$

$Y_{1}(0)=y_{0}$, 


$$
\begin{aligned}
d Y_{2}(t)= & Y_{2}(t)\left(-g+\frac{f}{A}-h Y_{2}(t)\right) d t \\
& -\beta Y_{2}(t) d w_{2}(t) \\
& +\int_{Z} Y_{2}(t) \eta(u) \widetilde{N}(d t, d u),
\end{aligned}
$$$$
Y_{2}(0)=y_{0} \text {. }
$$

By the comparison theorem of stochastic differential equation, we conclude that

$$
\begin{aligned}
& X_{1}(t) \leq x(t) \leq X_{2}(t), \\
& Y_{1}(t) \leq y(t) \leq Y_{2}(t)
\end{aligned}
$$

a.s.

$$
\begin{aligned}
& X_{1}(t)=\frac{\exp \left\{\left(a-c-\rho_{1}\right) t+\alpha w_{1}(t)+\kappa_{1}(t)\right\}}{x_{0}^{-1}+b \int_{0}^{t} \exp \left\{\left(a-c-\rho_{1}\right) s+\alpha w_{1}(s)+\kappa_{1}(s)\right\} d s}, \\
& X_{2}(t)=\frac{\exp \left\{\left(a-\rho_{1}\right) t+\alpha w_{1}(t)+\kappa_{1}(t)\right\}}{x_{0}^{-1}+b \int_{0}^{t} \exp \left\{\left(a-\rho_{1}\right) s+\alpha w_{1}(s)+\kappa_{1}(s)\right\} d s}, \\
& Y_{1}(t)=\frac{\exp \left\{\left(-g+f / A-\rho_{2}\right) t+\beta w_{2}(t)+\kappa_{2}(t)\right\}}{y_{0}^{-1}+\int_{0}^{t}\left(h+f / A^{2} X_{1}(t)\right) \exp \left\{\left(f / A-g-\rho_{2}\right) s+\beta w_{2}(s)+\kappa_{2}(s)\right\} d s}, \\
& Y_{2}(t)=\frac{\exp \left\{\left(-g+f / A-\rho_{2}\right) t+\beta w_{2}(t)+\kappa_{2}(t)\right\}}{y_{0}^{-1}+h \int_{0}^{t} \exp \left\{\left(f / A-g-\rho_{2}\right) s+\beta w_{2}(s)+\kappa_{2}(s)\right\} d s},
\end{aligned}
$$

where

$$
\begin{aligned}
\rho_{1} & =0.5 \alpha^{2}+\int_{Z}[\gamma(u)-\ln (1+\gamma(u))] \lambda(d u), \\
\rho_{2} & =0.5 \beta^{2}+\int_{Z}[\eta(u)-\ln (1+\eta(u))] \lambda(d u), \\
\kappa_{1}(t) & =\int_{0}^{t} \int_{Z} \ln (1+\gamma(u)) \widetilde{N}(d t, d u), \\
\kappa_{2}(t) & =\int_{0}^{t} \int_{Z} \ln (1+\eta(u)) \widetilde{N}(d t, d u) .
\end{aligned}
$$

Because $t \geq 0$ is the existence range of solutions $X_{1}(t), X_{2}(t), Y_{1}(t), Y_{2}(t)$, that means $T=+\infty$.

Next, we will show the asymptotic property of the solution to system (4).

Theorem 2. The solutions of system (4) are bounded in mean.

Proof. Let $V_{1}:=V_{1}(t, x)=e^{t} x^{p}(p>0)$. Direct computation, by the formula $E V_{1}(t, x(t))-E V_{1}\left(0, x_{0}\right)=E \int_{0}^{t} L V(s) d s[18]$, now leads to

$$
E\left(e^{t} x^{p}\right)=x^{p}(0)+E \int_{0}^{t} L V_{1} d s
$$

where

$$
\begin{aligned}
& L V_{1}=e^{t} x^{p}+e^{t} p x^{p}\left[a-b x+0.5(p-1) \alpha^{2}\right. \\
& \left.\quad-\frac{c y}{y+A x}+\int_{Z}\left[(1+\gamma(u))^{p}-1\right] \lambda(d u)\right] \leq e^{t} x^{p}
\end{aligned}
$$

$$
\begin{aligned}
& +e^{t} p x^{p}\left[a-b x+0.5(p-1) \alpha^{2}\right. \\
& \left.+\int_{Z}\left((1+\gamma(u))^{p}-1\right) \lambda(d u)\right] .
\end{aligned}
$$

From actual meanings of parameters and assumption $\left(H_{1}\right)$, we get that

$$
\begin{aligned}
x^{p} & +p x^{p}\left[a-b x+0.5(p-1) \alpha^{2}\right. \\
& \left.+\int_{Z}\left[(1+\gamma(u))^{p}-1\right] \lambda(d u)\right]
\end{aligned}
$$

is bounded. There exists a constant $K_{1}>0$, such that

$$
E e^{t} x^{p}(t) \leq x_{0}^{p}+\int_{0}^{t} K_{1} e^{s} d s
$$

when $1+x^{p}+p x^{p}\left[a-b x+0.5(p-1) \alpha^{2} \int_{Z}\left[(1+\gamma(u))^{p}-\right.\right.$ $1] \lambda(d u)]>0$; otherwise $\operatorname{Ee}^{t} x^{p}(t) \leq x_{0}^{p}$. Let $K_{2}=\max \left\{K_{1}, 0\right\}$; we have $E e^{t} x^{p}(t) \leq x_{0}^{p}+K_{2} e^{t}$. Denote $K=K_{2}+x_{0}^{p}$, then $E x^{p}(t) \leq K<+\infty$. Similarly, $E y^{p}(t) \leq K$.

\section{Persistence and Extinction}

In this section, some properties of the solutions of system (4) are investigated. Some sufficient conditions for persistence and extinction are shown. To proceed, some definitions and lemma are as follows.

Definition 3 (see [19]). (1) The population $x$ is called to be extinct if $x(t) \rightarrow 0(t \rightarrow \infty)$ a.s. 
(2) The population $x$ is called to be persistent if $\lim \inf _{t \rightarrow \infty}(1 / t) \int_{0}^{t} x(s) d s>0$ a.s.

(3) System (4) is called to be persistent if populations $x$ and $y$ are all persistent a.s.

Lemma 4 (see [20]). Under $\left(H_{1}\right)$ and $\left(H_{2}\right)$, suppose $Y(t) \in$ $C\left(\Omega \times[0,+\infty), R^{+}\right)$.

(1) If there exist three positive $T, k, k_{0}$ such that

$$
\begin{aligned}
\ln Y(t) \leq & k t-k_{0} \int_{0}^{t} Y(s) d s+\sum_{i=1}^{n} k_{i} w_{i}(t) \\
& +\sum_{i=1}^{n} c_{i} \int_{0}^{t} \int_{Z} \ln (1+\gamma(u)) \widetilde{N}(d s, d u) \quad \text { a.s. }
\end{aligned}
$$

for all $t>T$, where $k_{i}, c_{i}$ are constants, then

$$
\limsup _{t \rightarrow \infty} \frac{1}{t} \int_{0}^{t} Y(s) d s \leq \frac{k}{k_{0}} \quad \text { a.s. }
$$

If $k<0$ and other conditions are the same, then $\lim _{t \rightarrow \infty} Y(t)=$ 0 a.s.

(2) If there exist three positive $T, k, k_{0}$ such that

$$
\begin{aligned}
\ln Y(t) \geq & k t-k_{0} \int_{0}^{t} Y(s) d s+\sum_{i=1}^{n} k_{i} w_{i}(t) \\
& +\sum_{i=1}^{n} c_{i} \int_{0}^{t} \int_{Z} \ln (1+\gamma(u)) \widetilde{N}(d s, d u) \quad \text { a.s. }
\end{aligned}
$$

for all $t>T$, where $k_{i}, c_{i}$ are constants, then

$$
\liminf _{t \rightarrow \infty} \frac{1}{t} \int_{0}^{t} Y(s) d s \geq \frac{k}{k_{0}} \quad \text { a.s. }
$$

Now, the main results about persistence and extinction of system (4) are as follows.

Theorem 5. (1) The populations $x$ and $y$ are extinct if $a-\rho_{1}<0$ a.s.

(2) The population $x$ is persistent and $y$ is extinct if $a-c-$ $\rho_{1}>0, f / A-g-\rho_{2}<0$ a.s.

(3) The populations $x$ and $y$ are persistent if $a-c-\rho_{1}>$ $0, f / A-g-\rho_{2}>0$ a.s.

Proof. (1) By using the Itô formula, the following formulas are hold:

$$
\begin{aligned}
d \ln x(t)= & \left(a-b x(t)-\frac{c y(t)}{y(t)+A x(t)}-\rho_{1}\right) d t \\
& +\alpha d w_{1}(t) \\
& +\int_{Z} \ln (1+\gamma(u)) \widetilde{N}(d t, d u), \\
d \ln y(t)= & \left(-g-h y(t)+\frac{f x(t)}{y(t)+A x(t)}-\rho_{2}\right) d t \\
& +\beta d w_{2}(t) \\
& +\int_{Z} \ln (1+\eta(u)) \widetilde{N}(d t, d u) .
\end{aligned}
$$

Calculated by integral, we have the following form:

$$
\begin{aligned}
\ln x(t)-\ln x(0)= & \left(a-\rho_{1}\right) t+\alpha w_{1}(t)-\int_{0}^{t} b x(s) d s \\
& -\int_{0}^{t} \frac{c y(s)}{y(s)+A x(s)} d s+\kappa_{1}(t) \\
\leq & \left(a-\rho_{1}\right) t-b \int_{0}^{t} x(s) d s \\
& +\alpha w_{1}(t)+\kappa_{1}(t) .
\end{aligned}
$$

Then by Lemma 4 , note that $a<\rho_{1}$, and therefore $x(t) \rightarrow$ $0(t \rightarrow \infty)$ (population $x$ is extinct) a.s. Then, for arbitrarily small $\varepsilon>0$, there exist a sufficiently large $T$, when $t>T$, and we have $x(t)<\varepsilon$ and

$$
\begin{aligned}
d \ln y(t) \leq & \left(-g-h y(t)+\varepsilon-\rho_{2}\right) d t+\beta d w_{2}(t) \\
& +\int_{Z} \ln (1+\eta(u)) \widetilde{N}(d t, d u),
\end{aligned}
$$

where $-g-\varepsilon-\rho_{2}<0$. It is clear that $y(t) \rightarrow 0(t \rightarrow \infty)$ (population $y$ is extinct) a.s. Thus (1) is correct.

(2) From (1), the following forms are clear.

$$
\begin{aligned}
\ln x(t)-\ln x(0)= & \left(a-\rho_{1}\right) t+\alpha w_{1}(t)-\int_{0}^{t} b x(s) d s \\
& -\int_{0}^{t} \frac{c y(s)}{y(s)+A x(s)} d s+\kappa_{1} \\
\leq & \left(a-\rho_{1}\right) t-b \int_{0}^{t} x(s) d s \\
& +\alpha w_{1}(t)+\kappa_{1}, \\
\ln x(t)-\ln x(0)= & \left(a-\rho_{1}\right) t+\alpha w_{1}(t)-\int_{0}^{t} b x(s) d s \\
& -\int_{0}^{t} \frac{c y(s)}{y(s)+A x(s)} d s+\kappa_{1} \\
\geq & \left(a-c-\rho_{1}\right) t-b \int_{0}^{t} x(s) d s \\
& +\alpha w_{1}(t)+\kappa_{1} .
\end{aligned}
$$

Then by Lemma 4, we have

$$
\begin{aligned}
\frac{a-c-\rho_{1}}{b} & \leq \liminf _{t \rightarrow \infty} \frac{1}{t} \int_{0}^{t} x(s) d s \\
& \leq \limsup _{t \rightarrow \infty} \frac{1}{t} \int_{0}^{t} x(s) d s \leq \frac{a-\rho_{1}}{b} \text { a.s. }
\end{aligned}
$$

Therefore the population $x$ is persistent in mean. For population $y$, we have

$$
\begin{aligned}
d \ln y(t) \leq & \left(-g-h y(t)+\frac{f}{A}-\rho_{2}\right) d t+\beta d w_{2}(t) \\
& +\int_{Z} \ln (1+\eta(u)) \widetilde{N}(d t, d u) .
\end{aligned}
$$


From Lemma 4 and condition $f / A-g-\rho_{2}<0$, we know that $y(t) \rightarrow 0(t \rightarrow \infty)$ (i.e., population $y$ is extinct) a.s.

(3) In view of above, we can conclude the following when $a-c-\rho_{1}>0, g / A-g-\rho_{2}>0$, and

$$
\begin{aligned}
\ln y(t)-\ln y(0) \geq & \left(-g+\frac{f}{A}-\rho_{2}\right) t \\
& -\left(h+\frac{f}{A^{2} X_{1 *}}\right) \int_{0}^{t} y(s) d s \\
& +\beta w_{2}(t)+\kappa_{2}(t) .
\end{aligned}
$$

Then, we have

$$
\begin{aligned}
\frac{f / A-g-\rho_{2}}{h+f / A^{2} X_{1 *}} & \leq \liminf _{t \rightarrow \infty} \frac{1}{t} \int_{0}^{t} y(s) d s \\
& \leq \limsup _{t \rightarrow \infty} \frac{1}{t} \int_{0}^{t} y(s) d s \\
& \leq \frac{f / A-g-\rho_{2}}{h}, \text { a.s., }
\end{aligned}
$$

where $X_{1 *}$ is the minimum of $X_{1}(t)>0$.

It is clear that population $y$ is persistent.

Remark 6. For (1) in Theorem 5, it implies that the population $x$ of extinction a.s. leads to the extinction of population $y$ a.s. As shown in Figure 2, the simulations also affirm this point. In Figure 2, we can see that population $x$ becomes extinct, and after a while, population $y$ becomes extinct.

\section{Numerical Simulations}

We will demonstrate our results with the help of numerical simulations by using the Euler-Maruyama scheme [21, 22]. In numerical simulation, randomly selected parameters are as follows $a=0.55, b=0.21, c=0.15, f=0.93, h=$ $0.11, A=1.23, \alpha=0.75, \beta=0.73, Z=(0,+\infty)$, and $\lambda(Z)=1$, with simulation time span $T=50$ and step size $\Delta t=$ $T / N$, where $N=2^{12}$. The initial data is $\left(x_{0}, y_{0}\right)=(0.3,0.11)$ in Figure 2, and others are $(3,1)$.

(1) As illustrated in Figure 1, we choose $\gamma(u)=$ 0.99, $\eta(u)=0.85$, then $\rho_{1}=a-0.5 \alpha^{2}-\int_{Z}(\gamma(u)-\ln (1+$ $\gamma(u))) \lambda(d u)=0.58312, \rho_{2}=a-0.5 \beta^{2}-\int_{Z}(\eta(u)-\ln (1+$ $\eta(u))) \lambda(d u)=0.50126$, and $a-\rho_{1}=-0.03312<0, f / A-$ $g-\rho_{2}=-0.09517<0$. By Theorem 5 , the populations $x$ and $y$ are extinct a.s. Numerical experiments verify the correctness of (1) in Theorem 5.

(2) As illustrated in Figure 2, we choose $\gamma(u)=$ $0.99, \eta(u)=0.05$, then $\rho_{1}=a-0.5 \alpha^{2}-\int_{Z}(\gamma(u)-\ln (1+$ $\gamma(u))) \lambda(d u)=0.58312, \rho_{2}=a-0.5 \beta^{2}-\int_{Z}(\eta(u)-\ln (1+$ $\eta(u))) \lambda(d u)=0.26766$, and $a-\rho_{1}=-0.03312<0, f / A-g-$ $\rho_{2}=0.13844>0$. By Theorem 5 , the populations $x$ and $y$ are extinct a.s. Numerical experiments also verify the correctness of (1) in Theorem 5.

(3) As shown in Figure 3, we choose $\gamma(u)=0.15, \eta(u)=$ 0.91 , then $\rho_{1}=a-0.5 \alpha^{2}-\int_{Z}(\gamma(u)-\ln (1+\gamma(u))) \lambda(d u)=$ 0.29149, $\rho_{2}=a-0.5 \beta^{2}-\int_{Z}(\eta(u)-\ln (1+\eta(u))) \lambda(d u)=$

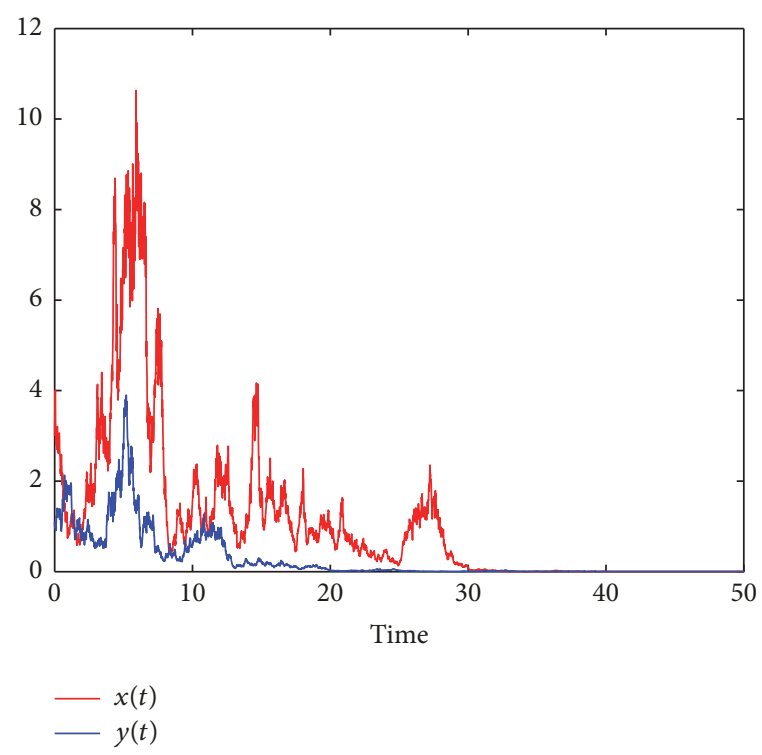

Figure 1

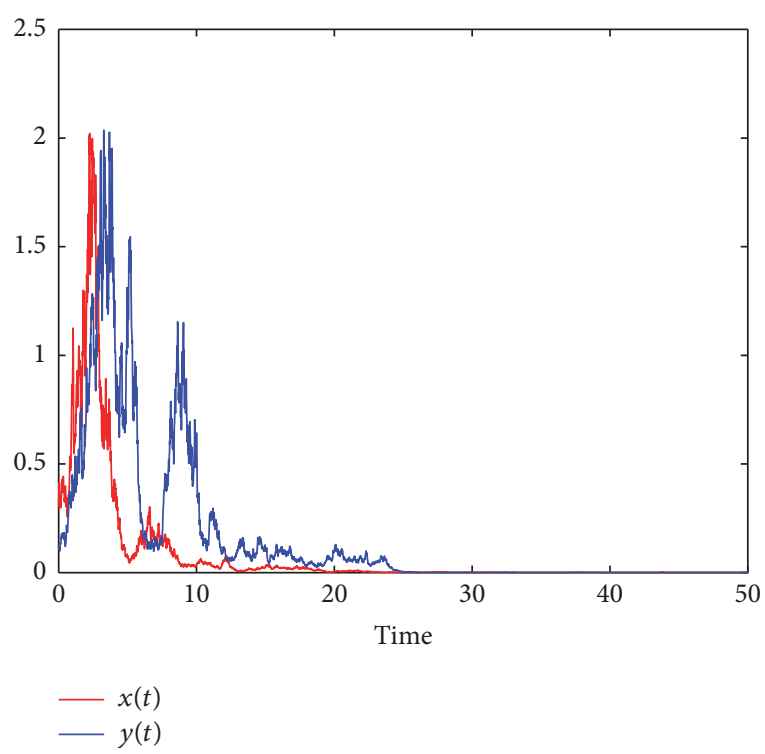

Figure 2

0.52935 , and $a-c-\rho_{1}=0.048512>0, f / A-g-\rho_{2}=$ $-0.12325<0$. By Theorem 5 , the population $x$ is persistent a.s. and population $y$ is extinct a.s. The correctness of (2) in Theorem 5 is verified.

(4) As shown in Figure 4, we choose $\gamma(u)=0.15, \eta(u)=$ 0.05 , then $\rho_{1}=a-0.5 \alpha^{2}-\int_{Z}(\gamma(u)-\ln (1+\gamma(u))) \lambda(d u)=$ 0.29149, $\rho_{2}=a-0.5 \beta^{2}-\int_{Z}(\eta(u)-\ln (1+\eta(u))) \lambda(d u)=$ 0.52935 , and $a-c-\rho_{1}=0.048512>0, f / A-g-\rho_{2}=$ $0.13844>0$. By Theorem 5, the populations $x$ and $y$ are persistent a.s. The validity of correctness of (3) in Theorem 5 is verified. 


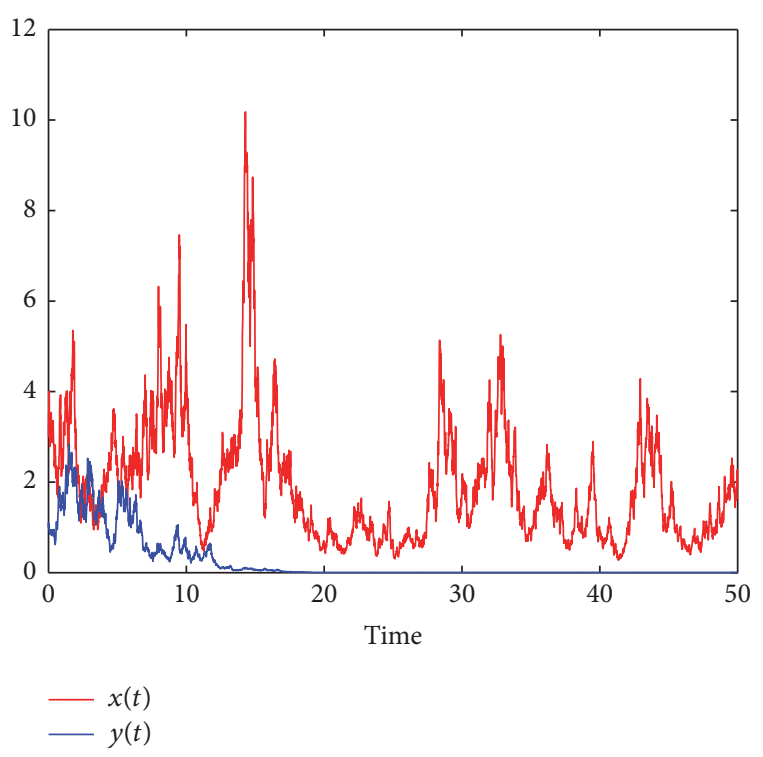

FIGURE 3

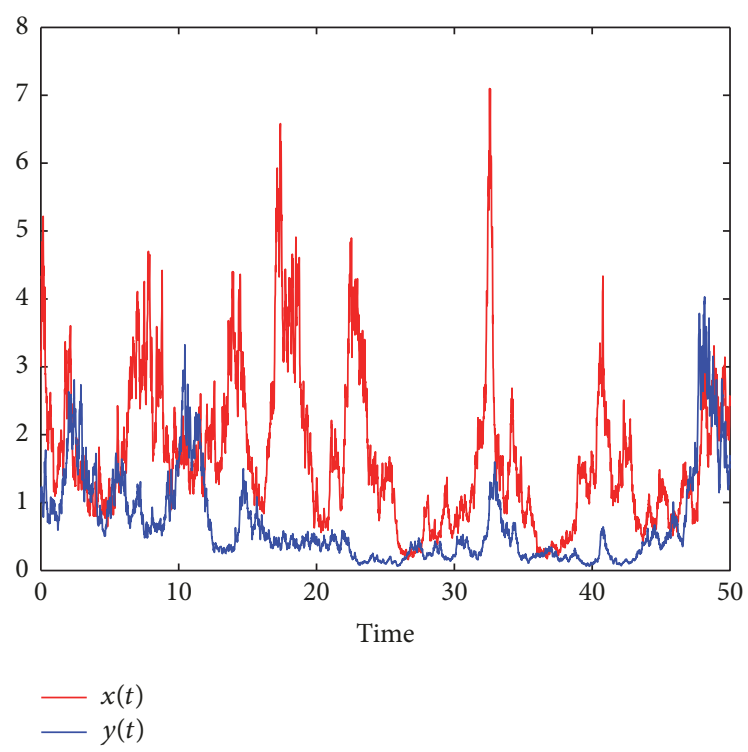

FIGURE 4

\section{Conclusions}

In this paper, a stochastic modified Bazykin system with Lévy jumps is discussed. Firstly, the existence of global positive solutions is investigated, and boundedness in mean is also given. Further, under related assumption, we obtain the sufficient conditions for the stochastic permanence and extinction of system (4). There are some interesting topics which deserve further discussion. For example, many authors consider the stationary distribution for stochastic predator-prey model with harvesting and delays [23-25], epidemic model with regime switching [26], impulsive stochastic model [27], and budworm growth model with Markovian switching [28]. The above investigations are left for future work.

\section{Conflicts of Interest}

The authors declare that there are no conflicts of interest regarding the publication of this manuscript.

\section{Acknowledgments}

This work is supported by National Natural Science Foundation of China (nos. 11771001, 11471015, and 11401002), Nature Science Foundation of Anhui Province (no. 1508085QA01 and no. 1508085MA10), Natural Science Foundation of Anhui Colleges (no. KJ2014A010), Program for Excellent Young Talents in University of Anhui Province (no. gxyq2017092), and Anhui Province Workshop of Prestigious Teacher (no. 2016msgzs006).

\section{References}

[1] A. D. Bazykin, Nonlinear Dynamics of Interacting Populations, vol. 11 of World Scientific Series on Nonlinear Science, Series A, World Scientific, River Edge, NJ, USA, 1998.

[2] M. Haque, "Ratio-dependent predator-prey models of interacting populations," Bulletin of Mathematical Biology, vol. 71, no. 2, pp. 430-452, 2009.

[3] Y. Kuang and E. Beretta, "Global qualitative analysis of a ratiodependent predator-prey system," Journal of Mathematical Biology, vol. 36, no. 4, pp. 389-406, 1998.

[4] D. Xiao and S. Ruan, "Global dynamics of a ratio-dependent predator-prey system," Journal of Mathematical Biology, vol. 43, no. 3, pp. 268-290, 2001.

[5] Y. Xiao and L. Chen, "Modeling and analysis of a predator-prey model with disease in the prey," Mathematical Biosciences, vol. 171, no. 1, pp. 59-82, 2001.

[6] S. Sarwardi, M. Haque, and P. . Mandal, "Ratio-dependent predator-prey model of interacting population with delay effect," Nonlinear Dynamics, vol. 69, no. 3, pp. 817-836, 2012.

[7] S. Sarwardi, M. Haque, and P. . Mandal, "Persistence and global stability of Bazykin predator-prey model with BEDdingtonDeAngelis response function," Communications in Nonlinear Science and Numerical Simulation, vol. 19, no. 1, pp. 189-209, 2014.

[8] M. Haque, "A detailed study of the Beddington-DeAngelis predator-prey model," Mathematical Biosciences, vol. 234, no. 1, pp. 1-16, 2011.

[9] J. Lv, K. Wang, and D. Chen, "Analysis on a stochastic twospecies ratio-dependent predator-prey model," Methodology and Computing in Applied Probability, vol. 17, no. 2, pp. 403-418, 2015.

[10] J. Bao and C. Yuan, "Stochastic population dynamics driven by Lévy noise," Journal of Mathematical Analysis and Applications, vol. 391, no. 2, pp. 363-375, 2012.

[11] M. Liu and C. Bai, "Dynamics of a stochastic one-prey twopredator model with Lévy jumps," Applied Mathematics and Computation, vol. 284, pp. 308-321, 2016.

[12] Q. Liu, D. Jiang, N. Shi, T. Hayat, and A. Alsaedi, "Stochastic mutualism model with Lévy jumps," Communications in Nonlinear Science and Numerical Simulation, vol. 43, pp. 78-90, 2017.

[13] W. Mao, L. Hu, and X. Mao, "The existence and asymptotic estimations of solutions to stochastic pantograph equations with diffusion and Lévy jumps," Applied Mathematics and Computation, vol. 268, pp. 883-896, 2015. 
[14] X. Zhang, D. Jiang, T. Hayat, and B. Ahmad, "Dynamics of a stochastic SIS model with double epidemic diseases driven by Lévy jumps," Physica A: Statistical Mechanics and its Applications, vol. 471, pp. 767-777, 2017.

[15] X. Meng and X. Wang, "Stochastic predator-prey system subject to Lévy jumps," Discrete Dynamics in Nature and Society, Article ID 5749892, 13 pages, 2016.

[16] Q. Zhu, "Razumikhin-type theorem for stochastic functional differential equations with Lévy noise and Markov switching," International Journal of Control, vol. 90, no. 8, pp. 1703-1712, 2017.

[17] L. Guo and Q. Zhu, "Stability analysis for stochastic VolterraLevin equations with Poisson jumps: fixed point approach," Journal of Mathematical Physics, vol. 52, no. 4, Article ID 042702, 042702, 15 pages, 2011.

[18] X. Mao, Stochastic Differential Equations and Their Applications, Horwood, Chichester, UK, 1997.

[19] M. Liu and K. Wang, "Dynamics of a two-prey one-predator system in random environments," Journal of Nonlinear Science, vol. 23, no. 5, pp. 751-775, 2013.

[20] M. Liu and K. Wang, "Stochastic Lotka-Volterra systems with Lévy noise," Journal of Mathematical Analysis and Applications, vol. 410, no. 2, pp. 750-763, 2014.

[21] D. J. Higham, X. Mao, and A. M. Stuart, "Strong convergence of Euler-type methods for nonlinear stochastic differential equations," SIAM Journal on Numerical Analysis, vol. 40, no. 3, pp. 1041-1063, 2002.

[22] L. Wang, Numerical Solutions of Stochastic Differential Equations, 2016.

[23] M. Liu, X. He, and J. Yu, "Dynamics of a stochastic regimeswitching predator-prey model with harvesting and distributed delays," Nonlinear Analysis: Hybrid Systems, vol. 28, pp. 87-104, 2018.

[24] H. Qiu and W. Deng, "Optimal harvesting of a stochastic delay tri-trophic food-chain model with Lévy jumps," Physica A: Statistical Mechanics and its Applications, vol. 492, pp. 1715-1728, 2018.

[25] J. Yu and M. Liu, "Stationary distribution and ergodicity of a stochastic food-chain model with Lévy jumps," Physica A: Statistical Mechanics and its Applications, vol. 482, pp. 14-28, 2017.

[26] X. Zhang, D. Jiang, A. Alsaedi, and T. Hayat, "Stationary distribution of stochastic SIS epidemic model with vaccination under regime switching," Applied Mathematics Letters, vol. 59, pp. 87-93, 2016.

[27] M. Liu, C. Du, and M. Deng, "Persistence and extinction of a modified Leslie-Gower Holling-type II stochastic predator-prey model with impulsive toxicant input in polluted environments," Nonlinear Analysis: Hybrid Systems, vol. 27, pp. 177-190, 2018.

[28] M. Liu and Y. Zhu, "Stability of a budworm growth model with random perturbations," Applied Mathematics Letters, vol. 79, pp. 13-19, 2018. 


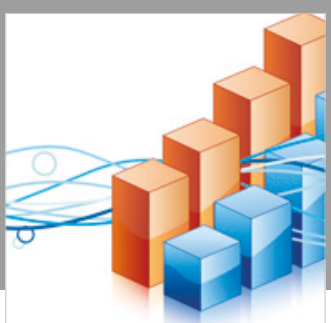

Advances in

Operations Research

\section{-n-m}
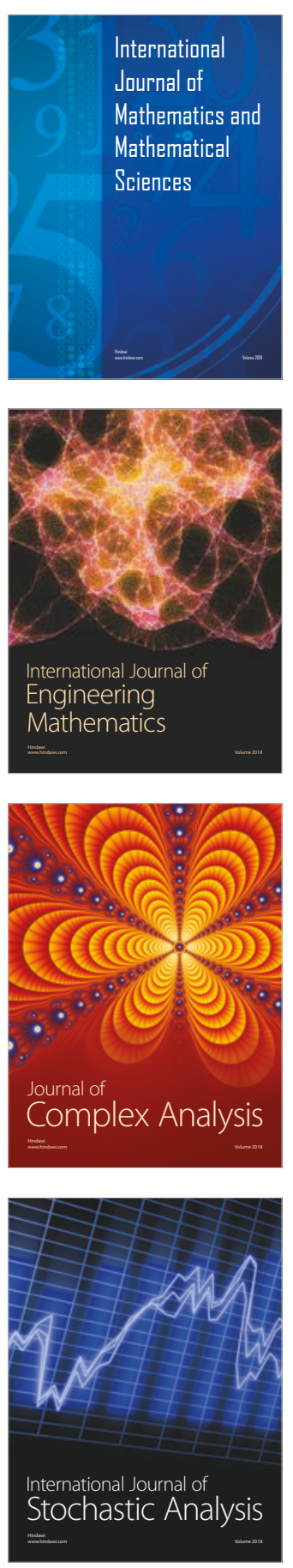
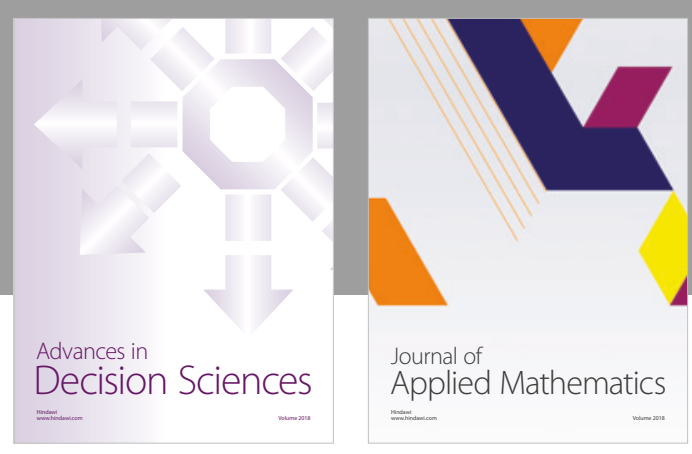

Journal of

Applied Mathematics
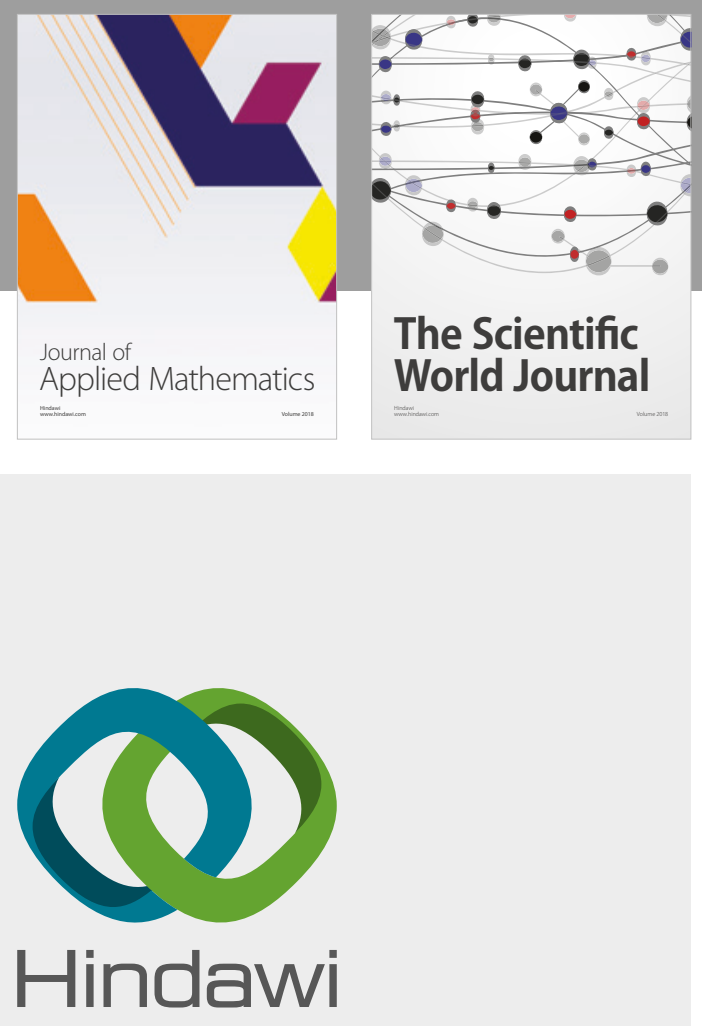

Submit your manuscripts at

www.hindawi.com

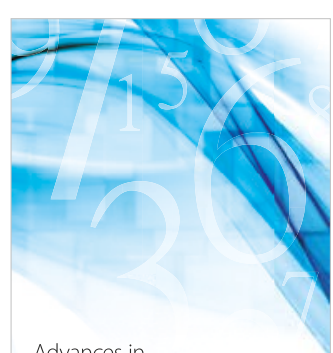

Advances in
Numerical Analysis
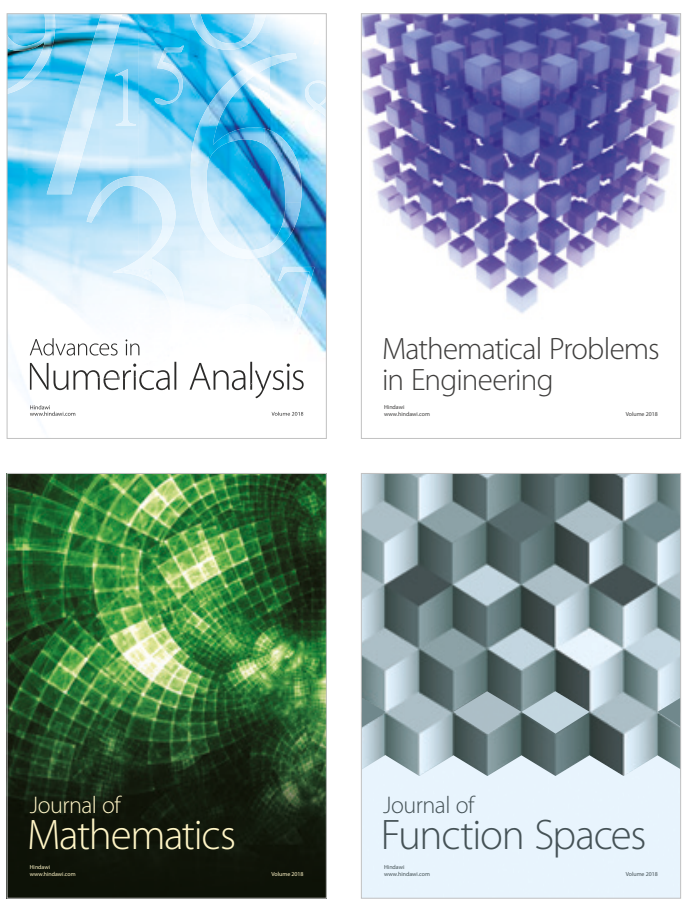

Mathematical Problems in Engineering

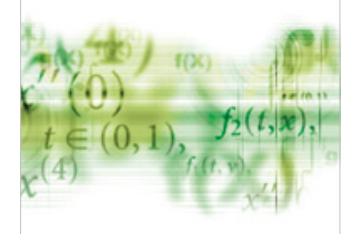

International Journal of

Differential Equations

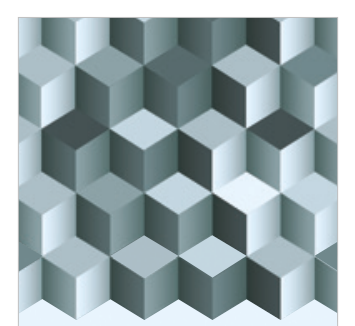

Journal of

Function Spaces

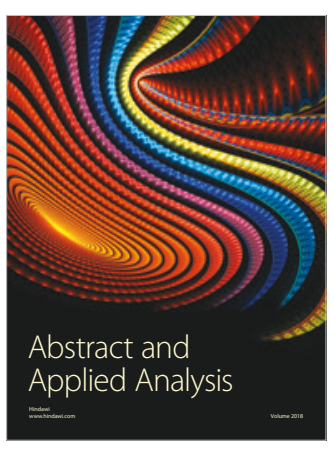

The Scientific

World Journal

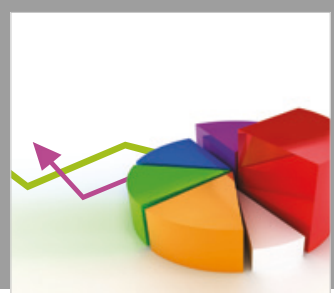

Journal of

Probability and Statistics
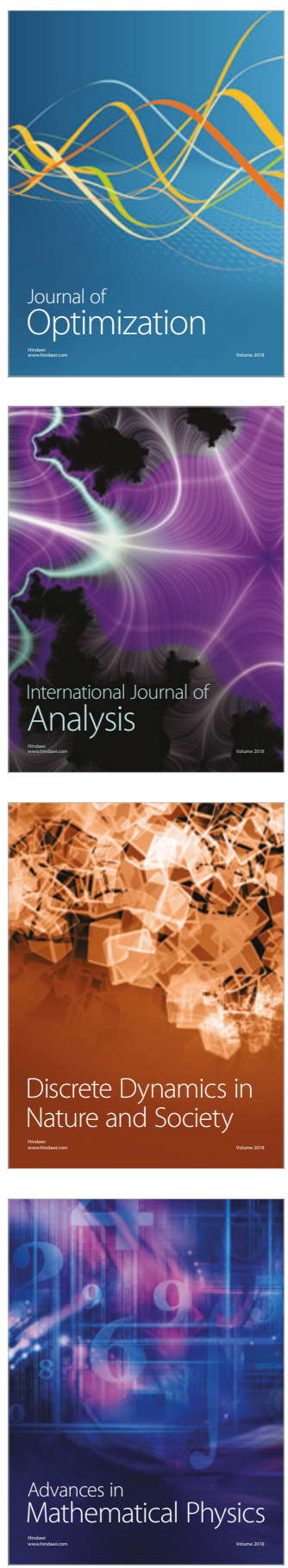